\title{
Robust Optimization via Probabilistic Cutting Plane Technique
}

\author{
Takayuki Wada ${ }^{1,2}$ and Yasumasa Fujisaki ${ }^{3}$ \\ ${ }^{1}$ Graduate School of Science and Technology, Kobe University \\ Nada, Kobe, 657-8501, JAPAN \\ E-mail: wada@cs23.cs.kobe-u.ac.jp \\ 2 JSPS Research Fellow (DC) \\ ${ }^{3}$ Department of Computer Science and Systems Engineering, Kobe University \\ Nada, Kobe, 657-8501, JAPAN \\ E-mail: fujisaki@cs.kobe-u.ac.jp
}

\begin{abstract}
A randomized algorithm is proposed for solving a robust optimization which is to minimize a convex objective function subject to a parameter dependent convex constraint. The probabilistic analytic center cutting plane method is employed for solving a series of robust feasibility problems defined by an optimality cut which corresponds to a provisional optimal value, where the parameter is randomly sampled in each iteration and the cut is sequentially updated for optimization. The algorithm always stops in a finite number of iterations, and finds a suboptimal solution and a suboptimal value. The suboptimal solution satisfies the constraint with a given probability and with a given probabilistic confidence, while the suboptimal value ensures that the feasibility set whose objective function is less than this value is too small to contain a ball with a given radius. The upper bounds of the numbers of random samples and updates of the algorithm are of polynomial order of the problem size.
\end{abstract}

\section{Introduction}

Robust convex optimization [1-4] is to minimize a convex objective function subject to a parameter dependent convex constraint. It gives a very useful framework for robustness analysis and synthesis of systems and control. In fact, several robust control problems can be recast as a robust convex optimization by using linear matrix inequality (LMI) technique $[5,6]$. However, solving the robust optimization needs a heavy computational effort. This is because its constraint depends on the uncertain parameter and thus we have to handle an infinite number of constraints.

In order to cope with this difficulty, several randomized algorithms [7] have been investigated, where stochastic gradient/ellipsoid methods for robust feasibility problems are incorporated [8-10]. In particular, the authors have proposed a randomized algorithm [10] which employs an optimality cut as well as a stochastic ellipsoid method. In contrast to an algorithm utilizing a penalty function [8], this algorithm has an explicit stopping rule, and thus it gives a solution within a finite number of iterations. Furthermore, the upper bounds of the numbers of random samples and updates are less than those of the stochastic bisection method [9].
The objective of this paper is to develop a sophisticated randomized algorithm for robust optimization which utilizes the optimality cut and a probabilistic analytic center cutting plane method. Note that the method has been used only for robust feasibility problems [11], where standard analytic center cutting plane methods $[12,13]$ are reformulated via randomization technique.

In this paper, we employ the probabilistic analytic center cutting plane method for solving a series of robust feasibility problems defined by an optimality cut which corresponds to a provisional optimal value, where we utilize a random sample of the uncertain parameter in each iteration and update the cut sequentially. This algorithm always stops in a finite number of iterations, and finds a suboptimal solution and a suboptimal value. Its significant features are as follows:

(i) With a specified probabilistic confidence, the suboptimal solution satisfies the constraint with a given high probability.

(ii) The suboptimal value ensures that the feasibility set whose objective function is less than this value is too small to contain a ball with a given radius.

(iii) The upper bounds of the numbers of random samples and updates of the algorithm are of polynomial order of the problem size.

This paper is organized as follows. In Section 2, the robust convex optimization is formally introduced. Then, in Section 3, a randomized algorithm based on the analytic center is presented, where its sample complexity is investigated. Subsequently, in Section 4, its sample complexity is further clarified in a practical situation, where the analytic center cannot be computed exactly in each iteration. In Section 5 , a modification of the stopping rule of the proposed algorithm is presented. This could reduce the sample complexity, though an LP should be solved in each iteration. Finally, in Section 6, some concluding remarks are provided. The proof of the main result is given in the appendix.

\section{Problem Formulation}

Let us consider a robust convex optimization

$$
\underset{z}{\operatorname{Minimize}} f(z) \quad \text { s.t. } g(z, \delta) \leq 0 \quad \forall \delta \in \Delta
$$


where $z \in \mathbb{R}^{n}$ is the optimization variable, $\delta \in \Delta \subseteq \mathbb{R}^{m}$ is the uncertain parameter, $\Delta \subseteq \mathbb{R}^{m}$ is the uncertainty set, $f(z)$ is the objective function which is convex in $z$, and $g(z, \delta)$ is the constraint which is measurable and convex in $z$ but can be nonlinear in $\delta$.

Now, let us introduce an upper bound $\gamma$ of the objective function. We consider a robust feasibility problem with optimization level $\gamma$

$$
\text { Find } z \text { s.t. } v(z, \delta, \gamma) \leq 0 \quad \forall \delta \in \Delta,
$$

where

$$
v(z, \delta, \gamma) \doteq\left[(\max \{0, f(z)-\gamma\})^{2}+(\max \{0, g(z, \delta)\})^{2}\right]^{1 / 2} .
$$

Notice that $v(z, \delta, \gamma) \leq 0$ is equivalent to $g(z, \delta) \leq 0$ and $f(z) \leq \gamma$. Thus, instead of solving the problem (1) directly, we minimize an upper bound $\gamma$ such that the robust feasibility problem with $\gamma$ is feasible. That is, we minimize $\gamma$ such that the corresponding set of $z$

$$
S(\gamma) \doteq\left\{z \in \mathbb{R}^{n}: v(z, \delta, \gamma) \leq 0, \forall \delta \in \Delta\right\}
$$

is not empty.

However, it is still difficult to find a solution of the problem (2) since this problem has a parameter dependent constraint. We therefore take a probabilistic approach [7], that is, we introduce a probability measure $\mathcal{P}$ on $\Delta$ and we seek a suboptimal solution based on random samples from $\Delta$.

\section{Optimization Based on Exact Analytic Center}

We first prepare parameters used in the algorithm. We set $r_{\mathrm{M}}>0$ and $z_{0} \in \mathbb{R}^{n}$ which describe an initial polytope

$$
C_{0} \doteq\left\{z \in \mathbb{R}^{n}:-r_{\mathrm{M}} \leq e_{i}^{\mathrm{T}}\left(z-z_{0}\right) \leq r_{\mathrm{M}}, i=1,2, \ldots, n\right\}
$$

where $e_{i}$ is the vector whose $i$ th element is 1 and the others are 0 . We treat the problem (1) within this initial polytope whose center $z_{0}$ is an initial candidate of the optimal solution. We also choose an initial candidate $\gamma_{0}$ of the optimal value and the tolerable error $r>0$ of the optimal value.

In the following algorithm, $\ell$ is the number of updates of $z_{\ell}$, and $k$ indicates that the candidate $z_{\ell}$ of the solution has not been updated for consecutive $k$ random samples. Then, $z_{\ell}$ is sequentially updated following the analytic center cutting plane method if $z_{\ell}$ and $\gamma_{\ell}$ do not satisfy $v\left(z_{\ell}, \delta, \gamma_{\ell}\right) \leq 0$ for a random sample $\delta$, where a barrier function $F_{\ell}(z)$ is also computed and updated. On the other hand, $\gamma_{\ell}$ is decreased with $r$ and the provisional optimal solution $\hat{z}$ and the provisional optimal value $\hat{\gamma}$ are updated if $z_{\ell}$ and $\gamma_{\ell}$ satisfy $v\left(z_{\ell}, \delta, \gamma_{\ell}\right) \leq 0$ for consecutive $\bar{k}$ random samples. When $\ell$ reaches $\bar{\ell}$, the algorithm stops with $\hat{z}$ and $\hat{\gamma}$ as output.

\section{Algorithm 1}

1. Select $\bar{k} \in \mathbb{N}$ and $\bar{\ell} \in \mathbb{N}$. Set $k:=0, \ell:=0, \hat{z}:=$ Null, $\hat{\gamma}:=$ Null, and

$$
F_{0}(z):=-\sum_{i=1}^{n}\left(\ln \left(r_{\mathrm{M}}-e_{i}^{\mathrm{T}}\left(z-z_{0}\right)\right)+\ln \left(r_{\mathrm{M}}+e_{i}^{\mathrm{T}}\left(z-z_{0}\right)\right)\right) \text {. }
$$

2. Draw $\delta$ in the set $\Delta$ according to the probability measure $\mathcal{P}$ and set $k:=k+1$.

3. If $v\left(z_{\ell}, \delta, \gamma_{\ell}\right)>0$, then go to Step 6 .

4. If $k<\bar{k}$, then go to Step 2 .

5. Set

$$
\gamma_{\ell}:=f\left(z_{\ell}\right)-r, \quad \hat{\gamma}:=f\left(z_{\ell}\right), \quad \hat{z}:=z_{\ell} .
$$

6. Update

$$
\begin{aligned}
\gamma_{\ell+1} & :=\gamma_{\ell} \\
z_{\ell+1} & :=\arg \min _{z} F_{\ell+1}(z) \\
F_{\ell+1}(z) & :=F_{\ell}(z)-\ln \left(a_{\ell}^{\mathrm{T}}\left(z_{\ell}-z\right)\right),
\end{aligned}
$$

where $a_{\ell}$ is a subgradient of $v(z, \delta, \gamma)$ with respect to $z$ at $z_{\ell}, \gamma_{\ell}$, and $\delta$. Set $\ell:=\ell+1$.

7. If $\ell<\bar{\ell}$, then set $k:=0$, and go to Step 2 .

8. Stop the algorithm with $\hat{z}$ and $\hat{\gamma}$ as output.

We refer to [3] for the definition of subgradient. We remark that $z_{\ell} \doteq \arg \min _{z} F_{\ell}(z)$ is the analytic center of

$$
C_{\ell} \doteq C_{0} \cap\left\{z \in \mathbb{R}^{n}: a_{i}^{\mathrm{T}}\left(z-z_{i}\right) \leq 0, i=0,1, \ldots, \ell-1\right\} .
$$

When we select $\bar{l}$ and $\bar{k}$ appropriately, this algorithm has the following significant properties.

Theorem 1 Suppose that an accuracy $\alpha \in(0,1)$, a risk $\beta \in$ $(0,1)$, and a parameter $r_{\mathrm{m}}>0$ are given. Select

$$
\begin{aligned}
& \bar{\ell} \doteq\left[\max \left\{50 n, 8 n^{2}\left(\frac{r_{\mathrm{M}}}{r_{\mathrm{m}}}\right)^{104 / 25}\right\}\right] \\
& \bar{k} \doteq\left[\ln \frac{\bar{\ell}}{\beta} \mid \ln \frac{1}{1-\alpha}\right] .
\end{aligned}
$$

Then, Algorithm 1 has the following properties.

1. The number of updates of $z_{\ell}$ is $\bar{\ell}$.

2. The number of random samples drawn in $\Delta$ is less than or equal to $\bar{k} \bar{\ell}$.

3. If Algorithm 1 stops with $\hat{z} \neq$ Null and $\hat{\gamma} \neq$ Null, the probability that $\hat{z}$ and $\hat{\gamma}$ satisfy

$$
\mathcal{P}\{\delta \in \Delta: v(\hat{z}, \delta, \hat{\gamma}) \leq 0\} \leq 1-\alpha
$$

is less than or equal to $\beta$.

4. If Algorithm 1 stops with $\hat{z} \neq$ Null and $\hat{\gamma} \neq$ Null, the set $C_{0} \cap S(\hat{\gamma}-r)$ does not contain any open ball with radius $r_{\mathrm{m}}$ (If Algorithm 1 stops with $\hat{z}=$ Null and $\hat{\gamma}=$ Null, the set $C_{0} \cap S\left(\gamma_{0}\right)$ does not contain any open ball with radius $\left.r_{\mathrm{m}}\right)$.

The statements 1 and 2 of Theorem 1 show that the upper bounds of the numbers of random samples and updates of Algorithm 1 are of polynomial order of the problem size. In this sense, the algorithm is efficient. Note here that we can also generate a random sample in polynomial-time in 
the problem size $[14,15]$. The statement 3 shows that, when Algorithm 1 stops with $\hat{z}$ and $\hat{\gamma}$ as output, the risk such that $\hat{z}$ is not a suboptimal solution with the optimization level $\hat{\gamma}$ and the accuracy $\alpha$ is less than or equal to $\beta$. That is, we can obtain a reliable solution if we set $\alpha$ and $\beta$ as small numbers. The statement 4 indicates the meaning of the suboptimality of $\hat{\gamma}$, i.e., the intersection of $C_{0}$ and $S(\hat{\gamma}-r)$ is too small to contain any open ball with radius $r_{\mathrm{m}}$.

\section{Optimization Based on $v$-Approximate Analytic Center}

In the previous section, we have assumed that we can compute the optimal solution of this problem

$$
z_{\ell+1}:=\arg \min _{z \in \mathbb{R}^{n}} F_{\ell+1}(z)
$$

at Step 6 of Algorithm 1, though this problem is approximately solved in practical situations. In this section, we consider a randomized algorithm which uses an approximate analytic center instead of the exact analytic center. To this end, we introduce the $v$-approximate analytic center $\tilde{z} \in \mathbb{R}^{n}$ of $C_{\ell}$ which is an element $\tilde{z} \in \operatorname{int}\left(C_{\ell}\right)$ such that

$$
\left(g_{\ell}(\tilde{z})\right)^{\mathrm{T}}\left(H_{\ell}(\tilde{z})\right)^{-1}\left(g_{\ell}(\tilde{z})\right) \leq v^{2}
$$

where $v \in[0,1)$ represents its accuracy, $\operatorname{int}(C)$ denotes the interior of the set $C$, and $g_{\ell}(z)$ and $H_{\ell}(z)$ are the gradient and the Hessian of $F_{\ell}(z)$, respectively. For simplicity, we will write $\tilde{g}_{\ell} \doteq g_{\ell}(\tilde{z})$ and $\widetilde{H}_{\ell} \doteq H_{\ell}(\tilde{z})$. Notice that $v$ gives a distance between the exact analytic center and this approximate center since $g_{\varepsilon}(z)$ at the exact analytic center is equal to 0 due to first order optimality conditions.

Algorithm 2 We replace Step 6 of Algorithm 1 by the following procedure.

6. Update

$$
\begin{aligned}
\gamma_{\ell+1} & :=\gamma_{\ell} \\
z_{\ell+1} & \in\left\{\tilde{z} \in \operatorname{int}\left(C_{\ell+1}\right): \tilde{g}_{\ell+1}^{\mathrm{T}} \widetilde{H}_{\ell+1}^{-1} \tilde{g}_{\ell+1} \leq v^{2}\right\} \\
F_{\ell+1}(z) & :=F_{\ell}(z)-\ln \left(a_{\ell}^{\mathrm{T}}\left(z_{\ell}-z\right)\right),
\end{aligned}
$$

where $a_{\ell}$ is a subgradient of $v(z, \delta, \gamma)$ with respect to $z$ at $z_{\ell}, \gamma_{\ell}$, and $\delta$. Set $\ell:=\ell+1$.

Theorem 2 Suppose that an accuracy $\alpha \in(0,1)$, a risk $\beta \in$ $(0,1)$, a parameter $r_{\mathrm{m}}>0$, and an accuracy $v \in[0,1)$ of the analytic center are given. Select

$$
\begin{aligned}
& \bar{\ell} \doteq\left[\max \left\{50 n, 8 n^{2} \exp \left(\frac{104}{25} \ln \frac{r_{\mathrm{M}}}{r_{\mathrm{m}}}+4 v \eta\right)\right\}\right] \\
& \bar{k} \doteq\left[\ln \frac{\bar{\ell}}{\beta} \ln \frac{1}{1-\alpha}\right],
\end{aligned}
$$

where

$$
\eta \doteq \frac{1+v+\sqrt{(1-v)^{2}+4}}{2(1-v)} .
$$

Then, Algorithms 1 and 2 have the same properties.
That is, even if we utilize a $v$-approximate analytic center instead of the exact analytic center, sample complexity of Algorithm 2 is still polynomial in the problem size. Furthermore, Algorithms 1 and 2 have the same guarantees for the suboptimal solution $\hat{z}$ and the suboptimal value $\hat{\gamma}$.

\section{Stopping Rule Modification}

Employing a modified stopping rule which uses a posterior analysis of $C_{0} \cap S(\hat{\gamma}-r)$, we can further improve the proposed algorithm. That is, if we compute the Chebyshev center of $C_{\ell}$ at each update, we can directly know whether the current $C_{\ell}$ can contain a ball with radius $r_{m}$ or not.

Note that this modification does not change the basic properties of the algorithm. Although we have to solve an LP at each update for finding the Chebyshev center of $C_{\ell}$, the algorithm could stop within a small number of updates. This is because $\bar{\ell}$ of Theorem 2 is often a loose bound and could be too large.

The modified algorithm and its properties are as follows.

Algorithm 3 We replace Step 6 of Algorithm 2 by the following procedures.

6. Update

$$
\begin{aligned}
\gamma_{\ell+1} & :=\gamma_{\ell} \\
F_{\ell+1}(z) & :=F_{\ell}(z)-\ln \left(a_{\ell}^{\mathrm{T}}\left(z_{\ell}-z\right)\right),
\end{aligned}
$$

where $a_{\ell}$ is a subgradient of $v(z, \delta, \gamma)$ with respect to $z$ at $z_{\ell}, \gamma_{\ell}$, and $\delta$.

(a) Find the Chebyshev center of $C_{\ell+1}$ :

$$
\begin{aligned}
\underset{z_{\mathrm{cb}} \in \mathbb{R}^{n}, r_{\mathrm{cb}} \in \mathbb{R}}{\operatorname{Maximize}} & r_{\mathrm{cb}} \\
\text { s.t. } & r_{\mathrm{M}}+e_{i}^{\mathrm{T}}\left(z_{\mathrm{cb}}-z_{0}\right) \geq r_{\mathrm{cb}} \\
& r_{\mathrm{M}}-e_{i}^{\mathrm{T}}\left(z_{\mathrm{cb}}-z_{0}\right) \geq r_{\mathrm{cb}} \\
& a_{j}^{\mathrm{T}}\left(\tilde{z}_{j}-z_{\mathrm{cb}}\right) \geq r_{\mathrm{cb}} \\
& i=1,2, \ldots, n, \quad j=0,1, \ldots, \ell .
\end{aligned}
$$

(b) If $r_{\mathrm{cb}} \leq r_{\mathrm{m}}$, stop the algorithm with $\hat{z}$ and $\hat{\gamma}$ as output.

(c) Update $z_{\ell+1} \in \mathbb{R}^{n}$ such that

$$
z_{\ell+1} \in\left\{\tilde{z} \in \operatorname{int}\left(C_{\ell+1}\right): \tilde{g}_{\ell+1}^{\mathrm{T}} \widetilde{H}_{\ell+1}^{-1} \tilde{g}_{\ell+1} \leq v^{2}\right\} .
$$

Set $\ell:=\ell+1$.

Corollary 1 Suppose that an accuracy $\alpha \in(0,1)$, a risk $\beta \in(0,1)$, a parameter $r_{\mathrm{m}}>0$, and an accuracy $v \in[0,1)$ of the analytic center are given. Select $\bar{\ell}$ and $\bar{k}$ as (5) and (6), respectively. Then, Algorithms 2 and 3 have the same properties except for the statement 1 , where the corresponding statement of Algorithm 3 is as follows.

\section{The number of updates $z_{\ell}$ is less than or equal to $\bar{\ell}$.}

That is, the algorithm could stop before the number of updates reaches its upper bound if we check the volume of the corresponding feasibility set in each update. 


\section{Concluding Remarks}

In this paper, we have proposed a randomized algorithm for solving robust convex optimization. This algorithm utilizes the optimality cut as well as the probabilistic analytic center cutting plane method. We have shown that the upper bounds of the numbers of random samples and updates of the algorithm are of polynomial order of the problem size. We have also shown that the randomized algorithm work well even if it utilizes a $v$-approximate analytic center instead of the exact analytic center.

This means that the proposed algorithm is efficient in practical situations. Furthermore, we have shown that a modification of the stopping rule is possible, where we could expect a fast convergence.

Acknowledgments: This work was partially supported by the Grant-in-Aid for JSPS Fellows, 20.291 and the Grantin-Aid for Scientific Research (C), 20560418, Japan Society for the Promotion of Science.

\section{References}

[1] L. El Ghaoui, F. Oustry, and H. Lebret: Robust solutions to uncertain semidefinite programs; SIAM Journal on Optimization, Vol. 9, No. 1, pp. 33-52 (1998)

[2] A. Ben-Tal and A. S. Nemirovski: Robust convex optimization; Mathematics of Operations Research, Vol. 23, No. 4, pp. 769-805 (1998)

[3] A. Ben-Tal and A. S. Nemirovskii: Lectures on Modern Convex Optimization: Analysis, Algorithms, and Engineering Applications; Society for Industrial \& Applied Mathematics (2001)

[4] A. Ben-Tal and A. S. Nemirovski: Selected topics in robust convex optimization; Mathematical Programing, Vol. 112, No. 1, pp. 125-158 (2008)

[5] S. Boyd, L. E. Ghaoui, E. Feron, and V. Balakrishnan: Linear Matrix Inequalities in System and Control Theory, Vol. 15 of SIAM studies in applied mathematics; SIAM (1994)

[6] R. E. Skelton, T. Iwasaki, and K. M. Grigoriadis: A unified Algebraic Approach to Linear Control Design; Taylor \& Francis (1997)

[7] R. Tempo, G. Calafiore, and F. Dabbene: Randomized Algorithms for Analysis and Control of Uncertain Systems; Springer-Verlag (2004)

[8] V. B. Tadić, S. Meyn, and R. Tempo. Randomized algorithms for semi-infinite programming problems. G. Calafiore and F. Dabbene, editors, Probabilistic and Randomized Methods for Design under Uncertainty, pp. 243-261. Springer-Verlag, (2006)

[9] Y. Oishi: Polynomial-time algorithms for probabilistic solutions of parameter-dependent linear matrix inequalities; Automatica, Vol. 43, No. 3, pp. 538-545 (2007)
[10] T. Wada and Y. Fujisaki: Sequential randomized algorithms for robust optimization; Proceedings of the 46th IEEE Conference on Decision and Control, pp. 6190 6195 (2007)

[11] G. Calafiore and F. Dabbene: A probabilistic analytic center cutting plane method for feasibility of uncertain LMIs; Automatica, Vol. 43, No. 12, pp. 2022-2033 (2007)

[12] J.-L. Goffin and J.-P. Vial: Convex nondifferentiable optimization: A survey focused on the analytic center cutting plane method; Optimization Methods and Software, Vol. 17, No. 5, pp. 805-867 (2002)

[13] J. E. Mitchell: Polynomial interior point cutting plane methods; Optimization Methods and Software, Vol. 18, No. 5, pp. 507-534 (2003)

[14] G. Calafiore, F. Dabbene, and R. Tempo: Randomized algorithms for probabilistic robustness with real and complex structured uncertainty; IEEE Transactions on Automatic Control, Vol. 45, No. 12, pp. 2218-2235 (2000)

[15] G. Calafiore and F. Dabbene: A probabilistic framework for problems with real structured uncertainty in systems and control; Automatica, Vol. 38, No. 8, pp. 1265-1276 (2002)

[16] S. Boyd and L. Vandernberghe: Convex Optimization; Cambridge University Press (2004)

\section{Appendix: Proof of Theorem 2}

In this appendix, we give a proof of Theorem 2. Notice that the proof of Theorem 1 can immediately be obtained by setting $v=0$ in the proof of Theorem 2, while the proof of Corollary 1 follows the main part of the proof of Theorem 2 .

The proof of Theorem 2 needs a series of preliminary results. Let us write the barrier function $F_{\ell}(z)$ as

$$
F_{\ell}(z) \doteq F_{0}(z)-\sum_{i=0}^{\ell-1} \ln \left(a_{i}^{\mathrm{T}}\left(\tilde{z}_{i}-z\right)\right) .
$$

The vector $\tilde{z}_{i}$ is a $v$-approximate analytic center defined by $F_{i}(z)$ which satisfies $\tilde{g}_{i}^{\mathrm{T}} \widetilde{H}_{i}^{-1} \tilde{g}_{i} \leq v^{2}$, where $\tilde{g}_{i}$ and $\widetilde{H}_{i}$ are the gradient and the Hessian of $F_{i}(z)$ at $\tilde{z}_{i}$, respectively. In the following discussion, we assume that $\left\|a_{i}\right\|=1$ for $i=0,1, \ldots, \ell-1$ without loss of generality. In fact, if $\left\|a_{i}\right\| \neq 1$, we can rewrite (8) as

$$
F_{\ell}(z)=F_{0}(z)-\sum_{i=0}^{\ell-1} \ln \left(\frac{a_{i}^{\mathrm{T}}}{\left\|a_{i}\right\|}\left(\tilde{z}_{i}-z\right)\right)-\sum_{i=0}^{\ell-1} \ln \left\|a_{i}\right\| .
$$

Thus, the difference appears only in the constant term, which does not affect the following discussion related to lower/upper bounds of $F_{\ell}(z)$.

The barrier function $F_{\ell}(z)$ is a self-concordant function $\left[16\right.$, Section 9.6]. That is, for any given direction $d \in \mathbb{R}^{n}$,

$$
\left|\frac{\mathrm{d}^{3}}{\mathrm{~d} t^{3}} F_{\ell}(z+t d)\right| \geq 2\left(\frac{\mathrm{d}^{2}}{\mathrm{~d} t^{2}} F_{\ell}(z+t d)\right)^{3 / 2}, \quad z+t d \in C_{\ell},
$$


where $t \in \mathbb{R}$. This property leads to the following lemma.

Lemma 1 For any integer $\ell \geq 1$,

$$
\begin{aligned}
F_{\ell}(z) \geq & F_{\ell}\left(\tilde{z}_{\ell}\right)+\tilde{g}_{\ell}^{\mathrm{T}}\left(z-\tilde{z}_{\ell}\right)+\sqrt{\left(z-\tilde{z}_{\ell}\right)^{\mathrm{T}} \widetilde{H}_{\ell}\left(z-\tilde{z}_{\ell}\right)} \\
& -\ln \left(1+\sqrt{\left(z-\tilde{z}_{\ell}\right)^{\mathrm{T}} \tilde{H}_{\ell}\left(z-\tilde{z}_{\ell}\right)}\right),
\end{aligned}
$$

where $\tilde{z}_{\ell}$ is a $v$-approximate analytic center of $F_{\ell}$.

In fact, from its self-concordant property, we have

$$
\left|\frac{\mathrm{d}}{\mathrm{d} t}\left(\frac{\mathrm{d}^{2}}{\mathrm{~d} t^{2}} F_{\ell}(z+t d)\right)^{1 / 2}\right| \leq 1
$$

Then, integrating the above inequality with respect to $t$ on $[0,1]$ twice, we can obtain (9). The detail can be found in the book [16, Section 9.6.3].

The following lemma is taken from [11-13].

Lemma 2 For any integer $\ell \geq 1$,

$$
\frac{1}{2} \sum_{i=0}^{\ell-1} \ln \left(a_{i}^{\mathrm{T}} \widetilde{H}_{i}^{-1} a_{i}\right) \leq \frac{\ell}{2} \ln \left(\frac{8 n^{2}}{\ell} \ln \left(1+\frac{\ell}{8 n^{2}}\right)\right)+\ell \ln r_{\mathrm{M}} .
$$

The following lemma can be proved by standard differential calculus.

Lemma 3 If $\sqrt{\tilde{g}_{\ell}^{\mathrm{T}} \widetilde{H}_{\ell}^{-1} \tilde{g}_{\ell}} \leq v$,

$$
\frac{1+\sqrt{g_{\ell}^{\mathrm{T}} \widetilde{H}_{\ell}^{-1} \tilde{g}_{\ell}}+\sqrt{\left(1-\sqrt{\tilde{g}_{\ell}^{\mathrm{T}} \widetilde{H}_{\ell}^{-1} \tilde{g}_{\ell}}\right)^{2}+4}}{2\left(1-\sqrt{\tilde{g}_{\ell}^{\mathrm{T}} \widetilde{H}_{\ell}^{-1} \tilde{g}_{\ell}}\right)} \leq \eta,
$$

where $\eta$ is given by (7).

Then, we obtain a lower bound of $\min _{z \in \mathbb{R}^{n}} F_{\ell}(z)$.

Lemma 4 For any integer $\ell \geq 1$,

$$
\begin{aligned}
\min _{z \in \mathbb{R}^{n}} F_{\ell}(z) \geq & (2 n+\ell) \ln \frac{1}{r_{M}}-\ell v \eta \\
& -\frac{\ell}{2} \ln \left(\frac{8 n^{2}}{\ell} \ln \left(1+\frac{\ell}{8 n^{2}}\right)\right),
\end{aligned}
$$

where $\eta$ is given by (7).

Proof: From Lemma 1, we have

$$
\begin{aligned}
\min _{z \in \mathbb{R}^{n}} F_{\ell}(z)= & \min _{z \in \mathbb{R}^{n}}\left(F_{\ell-1}(z)-\ln \left(a_{\ell-1}^{\mathrm{T}}\left(\tilde{z}_{\ell-1}-z\right)\right)\right. \\
\geq & \min _{z \in \mathbb{R}^{n}}\left(F_{\ell-1}\left(\tilde{z}_{\ell-1}\right)+\tilde{g}_{\ell-1}^{\mathrm{T}}\left(z-\tilde{z}_{\ell-1}\right)\right. \\
& +\sqrt{\left(z-\tilde{z}_{\ell-1}\right)^{\mathrm{T}} \tilde{H}_{\ell-1}\left(z-\tilde{z}_{\ell-1}\right)} \\
& -\ln \left(1+\sqrt{\left(z-\tilde{z}_{\ell-1}\right)^{\mathrm{T}} \tilde{H}_{\ell-1}\left(z-\tilde{z}_{\ell-1}\right)}\right) \\
& -\ln \left(-a_{\ell-1}^{\mathrm{T}}\left(z-\tilde{z}_{\ell-1}\right)\right) .
\end{aligned}
$$

Notice here that

$$
\begin{aligned}
& \left|\tilde{g}_{\ell-1}^{\mathrm{T}}\left(z-\tilde{z}_{\ell-1}\right)\right| \\
& \quad \leq \sqrt{\tilde{g}_{\ell-1}^{\mathrm{T}} \widetilde{H}_{\ell-1}^{-1} \tilde{g}_{\ell-1}} \sqrt{\left(z-\tilde{z}_{\ell-1}\right)^{\mathrm{T}} \tilde{H}_{\ell-1}\left(z-\tilde{z}_{\ell-1}\right)} \\
& \quad\left|a_{\ell-1}^{\mathrm{T}}\left(z-\tilde{z}_{\ell-1}\right)\right| \\
& \quad \leq \sqrt{a_{\ell-1}^{\mathrm{T}} \tilde{H}_{\ell-1}^{-1} a_{\ell-1}} \sqrt{\left(z-\tilde{z}_{\ell-1}\right)^{\mathrm{T}} \tilde{H}_{\ell-1}\left(z-\tilde{z}_{\ell-1}\right)} .
\end{aligned}
$$

Thus, we can derive a lower bound of (11) as

$$
\begin{aligned}
\min _{z \in \mathbb{R}^{n}} F_{\ell}(z) \geq & \min _{z \in \mathbb{R}^{n}}\left(F_{\ell-1}\left(\tilde{z}_{\ell-1}\right)\right. \\
& -\sqrt{\tilde{g}_{\ell-1}^{\mathrm{T}} \widetilde{H}_{\ell-1}^{-1} \tilde{g}_{\ell-1}} \sqrt{\left(z-\tilde{z}_{\ell-1}\right)^{\mathrm{T}} \widetilde{H}_{\ell-1}\left(z-\tilde{z}_{\ell-1}\right)} \\
& +\sqrt{\left(z-\tilde{z}_{\ell-1}\right)^{\mathrm{T}} \widetilde{H}_{\ell-1}\left(z-\tilde{z}_{\ell-1}\right)} \\
& -\ln \left(1+\sqrt{\left(z-\tilde{z}_{\ell-1}\right)^{\mathrm{T}} \widetilde{H}_{\ell-1}\left(z-\tilde{z}_{\ell-1}\right)}\right) \\
& -\ln \sqrt{a_{\ell-1}^{\mathrm{T}} \tilde{H}_{\ell-1}^{-1} a_{\ell-1}} \\
& -\ln \sqrt{\left(z-\tilde{z}_{\ell-1}\right)^{\mathrm{T}} \widetilde{H}_{\ell-1}\left(z-\tilde{z}_{\ell-1}\right)} .
\end{aligned}
$$

We next define

$$
\begin{aligned}
\Phi_{\ell-1}(x) \doteq & F_{\ell-1}\left(\tilde{z}_{\ell-1}\right)+\left(1-\sqrt{\tilde{g}_{\ell-1}^{\mathrm{T}} \widetilde{H}_{\ell-1}^{-1} \tilde{g}_{\ell-1}}\right) x \\
& +\ln (1+x)-\ln \sqrt{a_{\ell-1}^{\mathrm{T}} \widetilde{H}_{\ell-1}^{-1} a_{\ell-1}}-\ln x,
\end{aligned}
$$

where $x \doteq \sqrt{\left(z-\tilde{z}_{\ell-1}\right)^{\mathrm{T}} \widetilde{H}_{\ell-1}\left(z-\tilde{z}_{\ell-1}\right)} \geq 0$. If we differentiate $\Phi_{\ell-1}$, we have

$$
\frac{\mathrm{d}}{\mathrm{d} x} \Phi_{\ell-1}(x)=1-\sqrt{\tilde{g}_{\ell-1}^{\mathrm{T}} \widetilde{H}_{\ell-1}^{-1} \tilde{g}_{\ell-1}}-\frac{1}{(1+x)}-\frac{1}{x} .
$$

Hence, we see that

$$
x_{\ell-1}^{*} \doteq \frac{1+\sqrt{\tilde{g}_{\ell-1}^{\mathrm{T}} \widetilde{H}_{\ell-1}^{-1} \tilde{g}_{\ell-1}}+\sqrt{\left(1-\sqrt{\tilde{g}_{\ell-1}^{\mathrm{T}} \widetilde{H}_{\ell-1}^{-1} \tilde{g}_{\ell-1}}\right)^{2}+4}}{2\left(1-\sqrt{\tilde{g}_{\ell-1}^{\mathrm{T}} \widetilde{H}_{\ell-1}^{-1} \tilde{g}_{\ell-1}}\right)}
$$

is the positive stationary point of $\Phi_{\ell-1}(x)$. In other words, $\Phi_{\ell-1}\left(x_{\ell-1}^{*}\right)=0$.

Substituting $x_{\ell-1}^{*}$ into the inequality (12) and employing Lemma 3, we obtain

$$
\begin{aligned}
\min _{z \in \mathbb{R}^{n}} F_{\ell}(z) \geq & F_{\ell-1}\left(\tilde{z}_{\ell-1}\right)-x_{\ell-1}^{*} \sqrt{\tilde{g}_{\ell-1}^{\mathrm{T}} \widetilde{H}_{\ell-1}^{-1} \tilde{g}_{\ell-1}} \\
& +x_{\ell-1}^{*}-\ln \left(1+x_{\ell-1}^{*}\right)-\ln \left(x_{\ell-1}^{*} \sqrt{a_{\ell-1}^{\mathrm{T}} \bar{H}_{\ell-1}^{-1} a_{\ell-1}}\right) \\
\geq & F_{\ell-1}\left(\tilde{z}_{\ell-1}\right)-x_{\ell-1}^{*} \sqrt{\tilde{g}_{\ell-1}^{\mathrm{T}} \widetilde{H}_{\ell-1}^{-1} \tilde{g}_{\ell-1}} \\
& -\frac{1}{2} \ln \left(a_{\ell-1}^{\mathrm{T}} \widetilde{H}_{\ell-1}^{-1} a_{\ell-1}\right) \\
\geq & F_{\ell-1}\left(\tilde{z}_{\ell-1}\right)-v x_{\ell-1}^{*}-\frac{1}{2} \ln \left(a_{\ell-1}^{\mathrm{T}} \widetilde{H}_{\ell-1}^{-1} a_{\ell-1}\right)
\end{aligned}
$$




$$
\begin{aligned}
& \geq \min _{z \in \mathbb{R}^{n}} F_{\ell-1}(z)-v x_{\ell-1}^{*}-\frac{1}{2} \ln \left(a_{\ell-1}^{\mathrm{T}} \widetilde{H}_{\ell-1}^{-1} a_{\ell-1}\right) \\
& \geq \min _{z \in \mathbb{R}^{n}} F_{0}(z)-v \sum_{i=0}^{\ell-1} x_{i}^{*}-\frac{1}{2} \sum_{i=0}^{\ell-1} \ln \left(a_{i}^{\mathrm{T}} \widetilde{H}_{i}^{-1} a_{i}\right) \\
& \geq 2 n \ln \frac{1}{r_{\mathrm{M}}}-\ell v \eta-\frac{1}{2} \sum_{i=0}^{\ell-1} \ln \left(a_{i}^{\mathrm{T}} \widetilde{H}_{i}^{-1} a_{i}\right) .
\end{aligned}
$$

The above and Lemma 2 imply the inequality (10).

On the other hand, under a certain assumption, we can obtain an upper bound of $\min _{z \in \mathbb{R}^{n}} F_{\ell}(z)$.

Lemma 5 Suppose that the set $C_{\ell}$ contains an open ball with radius $r_{\mathrm{m}}$. Then, for any integer $\ell \geq 0$,

$$
\min _{z \in \mathbb{R}^{n}} F_{\ell}(z) \leq(2 n+\ell) \ln \frac{1}{r_{\mathrm{m}}} .
$$

Proof: Suppose that $z_{\mathrm{c}}$ is the center of an open ball with radius $r_{\mathrm{m}}$ which is contained by $C_{0} \cap S(\hat{\gamma})$. Then, we have

$$
\begin{array}{rlrl}
r_{\mathrm{M}}+e_{i}^{\mathrm{T}} z_{0}+e_{i}^{\mathrm{T}} z_{\mathrm{c}} & >r_{\mathrm{m}} & & \\
r_{\mathrm{M}}-e_{i}^{\mathrm{T}} z_{0}-e_{i}^{\mathrm{T}} z_{\mathrm{c}} & >r_{\mathrm{m}}, & & i=1,2, \ldots, n \\
a_{j}^{\mathrm{T}}\left(\tilde{z}_{j}-z_{\mathrm{c}}\right)>r_{\mathrm{m}}, & j=0,1, \ldots, \ell-1 .
\end{array}
$$

We therefore see that

$$
\min _{z \in \mathbb{R}^{n}} F_{\ell}(z) \leq F_{\ell}\left(z_{\mathrm{c}}\right)<(2 n+\ell) \ln \frac{1}{r_{\mathrm{m}}}
$$

which completes the proof.

We state the following two lemmas without proofs.

Lemma 6 For $\ell \geq 50 n$,

$$
2 n+\ell \leq \frac{26}{25} \ell .
$$

Lemma 7 For any $x>0$,

$$
\frac{x}{\log (1+x)}>x^{1 / 2}
$$

Now, let us consider the proof of Theorem 2 .

Proof: The items 1 and 2 of Theorem 2 can be derived by counting the number of updates and iterations of Algorithm 2 directly.

The proof of the item 3 is similar to that of [9]. We first define two events.

$\mathcal{G}_{\ell}$ : The candidate $z_{\ell}$ with $\gamma_{\ell}$ whose number of updates reaches $\ell$ is not updated for consecutive $\bar{k}$ times.

$\mathcal{B}_{\ell}$ : The candidate $z_{\ell}$ with $\gamma_{\ell}$ whose number of updates reaches $\ell$ satisfies

$$
\mathcal{P}\left\{\delta \in \Delta: v\left(z_{\ell}, \delta, \gamma_{\ell}\right) \leq 0\right\} \leq 1-\alpha
$$

for a given accuracy $\alpha$.

Then, we see that

$$
\mathcal{P}^{\infty}\left\{\mathcal{G}_{\ell} \cap \mathcal{B}_{\ell}\right\} \leq \mathcal{P}^{\infty}\left\{\mathcal{G}_{\ell} \mid \mathcal{B}_{\ell}\right\} \leq(1-\alpha)^{\bar{k}}
$$

holds for any $\ell=0,1,2, \ldots, \bar{\ell}-1$, which implies that

$$
\mathcal{P}^{\infty}\left\{\bigcup_{\ell=0}^{\bar{\ell}-1}\left(G_{\ell} \cap \mathcal{B}_{\ell}\right)\right\} \leq \bar{\ell}(1-\alpha)^{\bar{k}} \leq \beta
$$

with $\bar{k}$ defined by (4). This gives the proof of the item 3 .

The item 4 of Theorem 2 is proved by using the lemmas prepared above. Suppose that the set $C_{\ell}$ contains an open ball with radius $r_{\mathrm{m}}$. From Lemmas 4 and 5, we have

$$
\begin{aligned}
& (2 n+\ell) \ln \frac{1}{r_{M}}-\ell v \eta-\frac{\ell}{2} \ln \left(\frac{8 n^{2}}{\ell} \ln \left(1+\frac{\ell}{8 n^{2}}\right)\right) \\
& \leq(2 n+\ell) \ln \frac{1}{r_{\mathrm{m}}} .
\end{aligned}
$$

However, this cannot hold if $\ell=\bar{\ell}$. In other words, the set $C_{\bar{\ell}}$ cannot contain any open ball with radius $r_{\mathrm{m}}$. This is because we have

$$
\begin{aligned}
& (2 n+\bar{\ell}) \ln \frac{1}{r_{\mathrm{M}}}-\bar{\ell} \nu \eta+\frac{\bar{\ell}}{2} \ln \left(\frac{\bar{\ell}}{8 n^{2}} / \ln \left(1+\frac{\bar{\ell}}{8 n^{2}}\right)\right) \\
& >(2 n+\bar{\ell}) \ln \frac{1}{r_{\mathrm{M}}}-\bar{\ell} v \eta+\frac{\bar{\ell}}{2} \ln \left(\frac{\bar{\ell}}{8 n^{2}}\right)^{1 / 2} \\
& =(2 n+\bar{\ell}) \ln \frac{1}{r_{\mathrm{M}}}-\bar{\ell} v \eta+\frac{\bar{\ell}}{4} \ln \frac{\bar{\ell}}{8 n^{2}} \\
& \geq(2 n+\bar{\ell}) \ln \frac{1}{r_{\mathrm{M}}}+\frac{26}{25} \bar{\ell} \ln \frac{r_{\mathrm{M}}}{r_{\mathrm{m}}} \\
& \geq(2 n+\bar{\ell}) \ln \frac{1}{r_{\mathrm{M}}}+(2 n+\bar{\ell}) \ln \frac{r_{\mathrm{M}}}{r_{\mathrm{m}}} \\
& =(2 n+\bar{\ell}) \ln \frac{1}{r_{\mathrm{m}}}
\end{aligned}
$$

if we employ Lemmas 6 and 7 with the definition (5) of $\bar{\ell}$. Since these inequalities (13) and (14) contradict each other, we can actually conclude that the set $C_{\bar{\ell}}$ can not contain any open ball with radius $r_{\mathrm{m}}$. Notice here that

$$
\begin{aligned}
C_{\ell+1} & \supseteq C_{\ell} \cap S\left(\gamma_{\ell}\right) \\
S\left(\gamma_{\ell+1}\right) & \subseteq S\left(\gamma_{\ell}\right)
\end{aligned}
$$

hold for any positive integer $\ell$. Then, it turns out that

$$
\begin{aligned}
C_{\bar{\ell}} \supseteq C_{\bar{\ell}-1} \cap S\left(\gamma_{\bar{\ell}-1}\right) \supseteq C_{\bar{\ell}-1} \cap S\left(\gamma_{\bar{\ell}}\right) \\
\quad \supseteq\left(C_{\bar{\ell}-2} \cap S\left(\gamma_{\bar{\ell}-2}\right)\right) \cap S\left(\gamma_{\bar{\ell}}\right) \supseteq C_{\bar{\ell}-2} \cap S\left(\gamma_{\bar{\ell}}\right) \\
\quad \supseteq \ldots \supseteq C_{0} \cap S\left(\gamma_{\bar{\ell}}\right) .
\end{aligned}
$$

Since $\gamma_{\bar{\ell}}=\hat{\gamma}-r$, we see that $C_{0} \cap S(\hat{\gamma}-r)$ can not contain any open ball with radius $r_{\mathrm{m}}$. 